\title{
The catalogue of radial velocity variable hot subluminous stars from the MUCHFUSS project (Corrigendum)
}

\author{
S. Geier ${ }^{1,2}$, T. Kupfer ${ }^{3}$, U. Heber ${ }^{2}$, V. Schaffenroth ${ }^{2,4}$, B. N. Barlow ${ }^{5}$, R. H. Østensen ${ }^{6}$, S. J. O’ Toole ${ }^{7}$, E. Ziegerer ${ }^{2}$,
} C. Heuser ${ }^{2}$, P. F. L. Maxted ${ }^{8}$, B. T. Gänsicke ${ }^{9}$, T. R. Marsh ${ }^{9}$, R. Napiwotzki ${ }^{10}$, P. Brünner ${ }^{2}$, M. Schindewolf ${ }^{2}$, and F. Niederhofer ${ }^{1}$

${ }^{1}$ European Southern Observatory, Karl-Schwarzschild-Str. 2, 85748 Garching, Germany e-mail: sgeier@eso.org

2 Dr. Karl Remeis-Observatory \& ECAP, Astronomical Institute, Friedrich-Alexander University Erlangen-Nuremberg, Sternwartstr. 7, 96049 Bamberg, Germany

3 Department of Astrophysics/IMAPP, Radboud University Nijmegen, PO Box 9010, 6500 GL Nijmegen, The Netherlands

${ }_{4}$ Institute for Astro- and Particle Physics, University of Innsbruck, Technikerstr. 25/8, 6020 Innsbruck, Austria

5 Department of Physics, High Point University, One University Parkway, High Point, NC 27268, USA

${ }^{6}$ Institute of Astronomy, KU Leuven, Celestijnenlaan 200D, 3001 Heverlee, Belgium

7 Australian Astronomical Observatory, PO Box 915, North Ryde NSW 1670, Australia

8 Astrophysics Group, Keele University, Staffordshire, ST5 5BG, UK

9 Department of Physics, University of Warwick, Conventry CV4 7AL, UK

10 Centre of Astrophysics Research, University of Hertfordshire, College Lane, Hatfield AL10 9AB, UK

A\&A 577, A26 (2015), DOI: 10.1051/0004-6361/201525666

Key words. binaries: spectroscopic - subdwarfs - stars: horizontal branch - stars: atmospheres - errata, addenda

The function we used to calculate the logarithm of the falsedetection probability provides the natural $\operatorname{logarithm} \ln p$ and not the decadal logarithm as incorrectly assumed in the paper. This mildy affects the number of radial velocity (RV) variable stars and significantly the number of RV variable candidates in our sample. The conclusions of the paper essentially remain the same.

We consider the detection of RV variability to be significant, if the false-detection probability $p$ is smaller than $0.01 \%$ $(\ln p<-9.2)$. The fraction of such significant detections in our initial sample of 196 is now $39 \%$ (76 objects). Objects with falsedetection probabilities between $0.01 \%$ and $5 \%(\ln p=-9.2$ to $\ln p=-3.0)$ are regarded as candidates for RV variability and constitute $27 \%$ of the initial sample (53 objects). About $34 \%$ ( $\ln p>-3.0,67$ objects) are regarded as non-detections. Removing those non-detections we end up with a sample of 129 stars, which show RV variability with probabilites between $95 \%$ and $99.9 \%$ (see Table 1 ).

Tables 3-5 and A.1 as well as Figures $1-6$ of the original paper have been updated (Tables 2-5, Figs. 1-4).

The corrected upper limit for the fraction of extremely close binary $\mathrm{sdB}+\mathrm{NS} / \mathrm{BH}$ binaries is $1.5 \%$ instead of $1.3 \%$.

The RV-variable sample now contains 18 helium-rich hot subdwarf stars. 6 of them show significant RV variations while 12 qualify as candidates (see Table 3). The He-sdOB J160450.44+051909.2 discussed in the paper is not regarded as RV-variable candidate any more.
Table 1. Sample statistics.

\begin{tabular}{llll}
\hline \hline Class & RV variable & $\begin{array}{l}\text { RV variable } \\
\text { candidates }\end{array}$ & $\begin{array}{l}\text { Non- } \\
\text { detections }\end{array}$ \\
\hline H-rich sdO/B & 65 & 36 & 51 \\
He-rich sdO/B & 6 & 12 & 11 \\
Others & 5 & 5 & 5 \\
\hline Total & 76 & 53 & 67 \\
\hline
\end{tabular}

\section{References}

Althaus, L. G., Panei, J. A., Miller Bertolami, M. M., et al. 2009, ApJ, 704, 1605

Dorman, B., Rood, R. T., \& O'Connell, R. W. 1993, ApJ, 419, 596

Driebe, T., Schönberner, D., Bloecker, T., \& Herwig, F. 1998, A\&A, 339, 123

Geier, S., Hirsch, H., Tillich, A., et al. 2011a, A\&A, 530, A28

Geier, S., Maxted, P. F. L., Napiwotzki, R., et al. 2011b, A\&A, 526, A39

Geier, S., Schaffenroth, V., Drechsel, H., et al. 2011c, ApJ, 731, L22

Kupfer, T., Geier, S., Schaffenroth, V., et al. 2015, A\&A, 576, A44

Østensen, R. H., Geier, S., Schaffenroth, V., et al. 2013, A\&A, 559, A35

Schaffenroth, V., Geier, S., Heber, U., et al. 2014, A\&A, 564, A98

Reindl, N., Geier, S., Kupfer, T., et al. 2016, A\&A, 587, A101

Schaller, G., Schaerer, D., Meynet, G., \& Maeder, A. 1992, A\&AS, 96, 269

Schönberner, D. 1983, ApJ, 272, 708

Werner, K., Rauch, T., \& Kepler, S. O. 2014, A\&A, 564, A53 

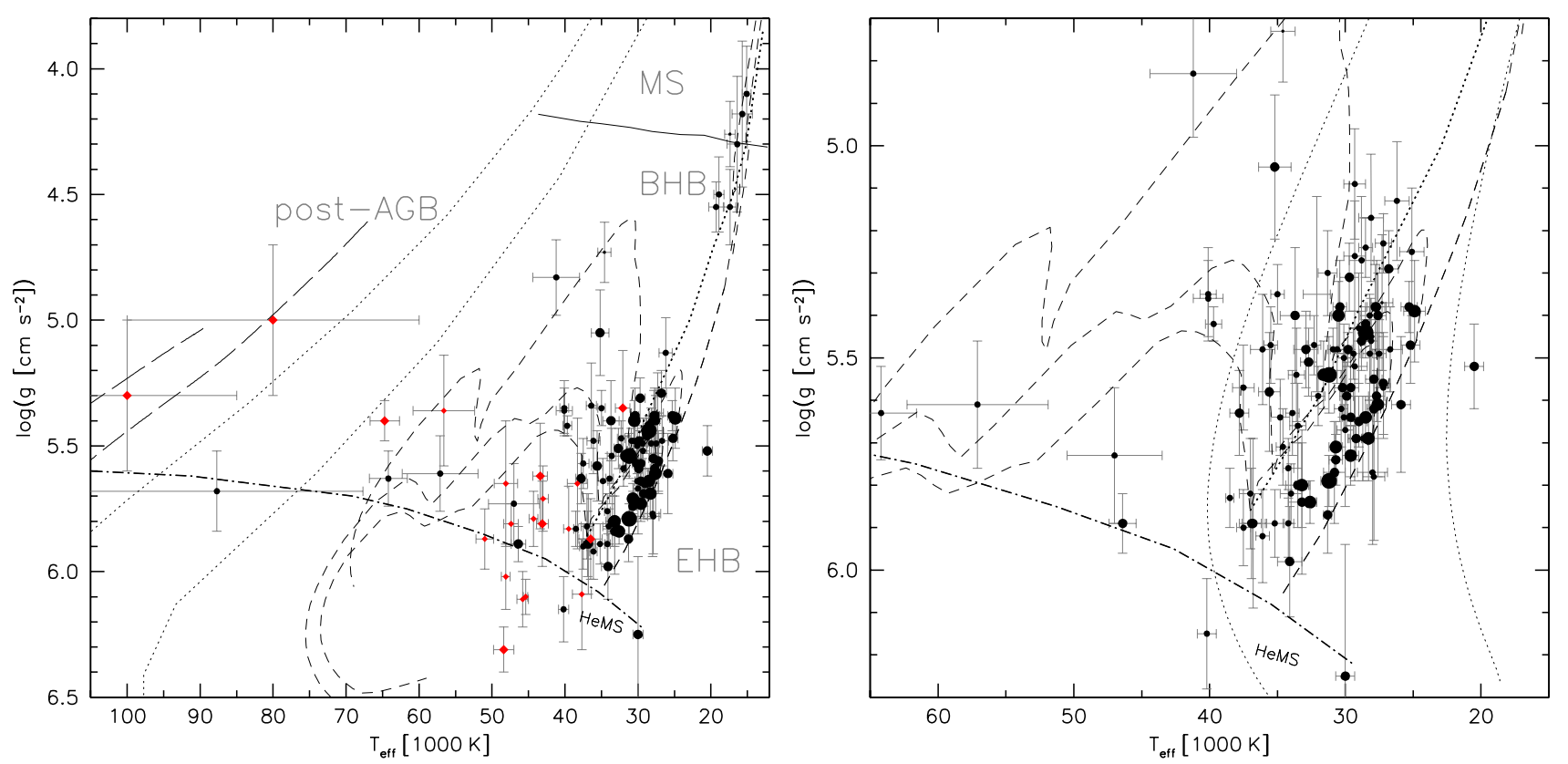

Fig. 1. Left panel: $T_{\text {eff }}-\log g$ diagram of the full sample of hot, subluminous, $\mathrm{RV}$-variable stars. The size of the symbols scales with $\Delta \mathrm{RV}_{\text {max }}$. The black circles mark stars with hydrogen dominated atmospheres $(\log y<0)$, while the red diamonds mark stars with helium dominated atmospheres. The helium main sequence (HeMS) and the HB band are superimposed with HB evolutionary tracks (dashed lines) for subsolar metallicity $(\log z=-1.48)$ from Dorman et al. (1993). The three tracks in the high temperature range correspond to helium core masses of 0.488 , 0.490 and $0.495 M_{\odot}$ (from bottom-left to top-right). Those tracks mark the EHB evolution, since the stars do not reascend the giant branch in the helium shell-burning phase. The two tracks in the upper right correspond to core masses of 0.53 and $0.54 M_{\odot}$. Blue horizontal branch stars following those tracks are expected to experience a second giant phase. The solid line marks the relevant part of the zero-age main sequence for solar metallicity taken from Schaller et al. (1992). The two dotted lines are post-AGB tracks for hydrogen-rich stars with masses of 0.546 (lower line) and $0.565 M_{\odot}$ (upper line) taken from Schönberner (1983). The two long-dashed lines are post-AGB tracks for helium-rich stars with masses of 0.53 (lower line) and $0.609 M_{\odot}$ (upper line) taken from Althaus et al. (2009). Right panel: $T_{\text {eff }}-\log g$ diagram of RV variable hydrogen-rich sdB and sdOB stars. The two dotted lines mark post-RGB tracks (Driebe et al. 1998) for core masses of 0.234 (left) and 0, $259 M_{\odot}$ (right).
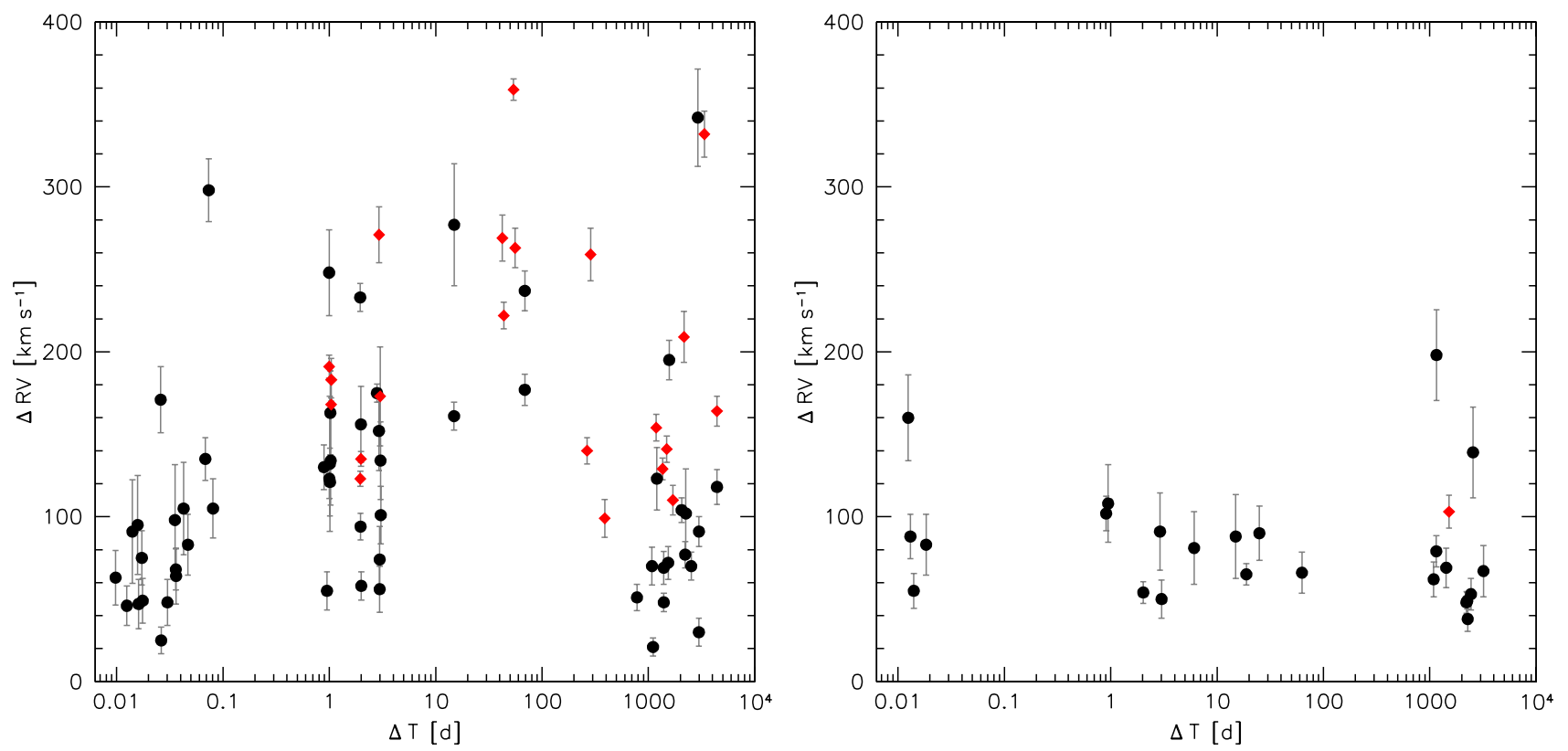

Fig. 2. Left panel: highest RV shift between individual spectra plotted against time difference between the corresponding observing epochs. The filled red diamonds mark sdB binaries with known orbital parameters (Kupfer et al. 2015), while the filled black circles mark the rest of the hydrogen-rich sdB sample of RV variable stars. Right panel: the same plot for the hydrogen-rich sdOB and sdO sample of RV variable stars. 
S. Geier et al.: The catalogue of radial velocity variable hot subluminous stars from the MUCHFUSS project (Corrigendum)
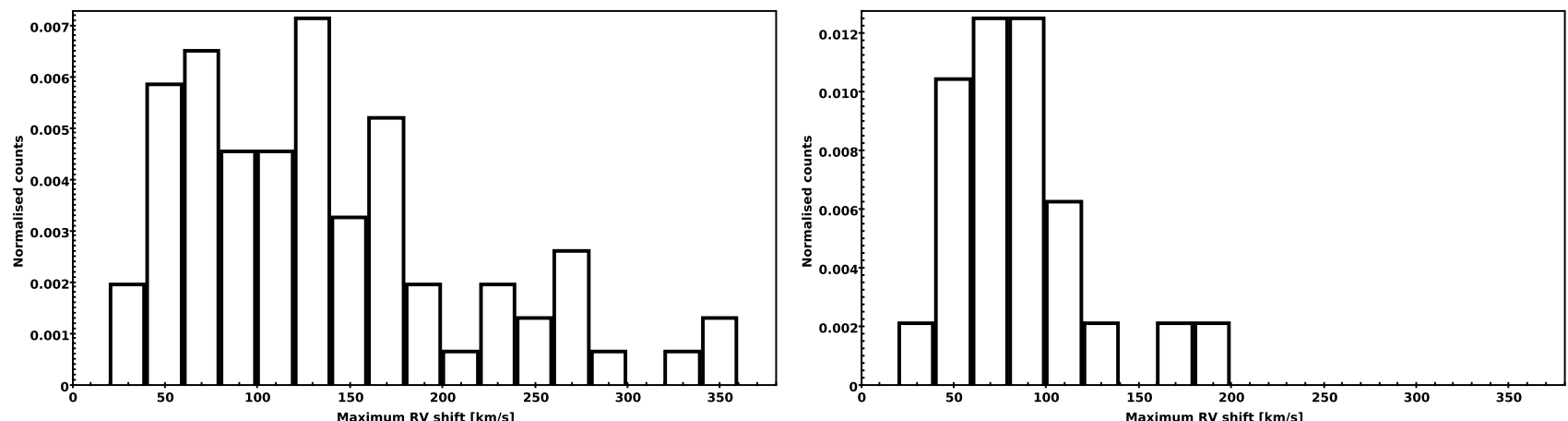

Fig. 3. $\Delta \mathrm{RV}_{\max }$ distribution of $\mathrm{RV}$-variable sdB stars (left panel) as well as sdOB and sdO stars with hydrogen-rich atmospheres (right panel).
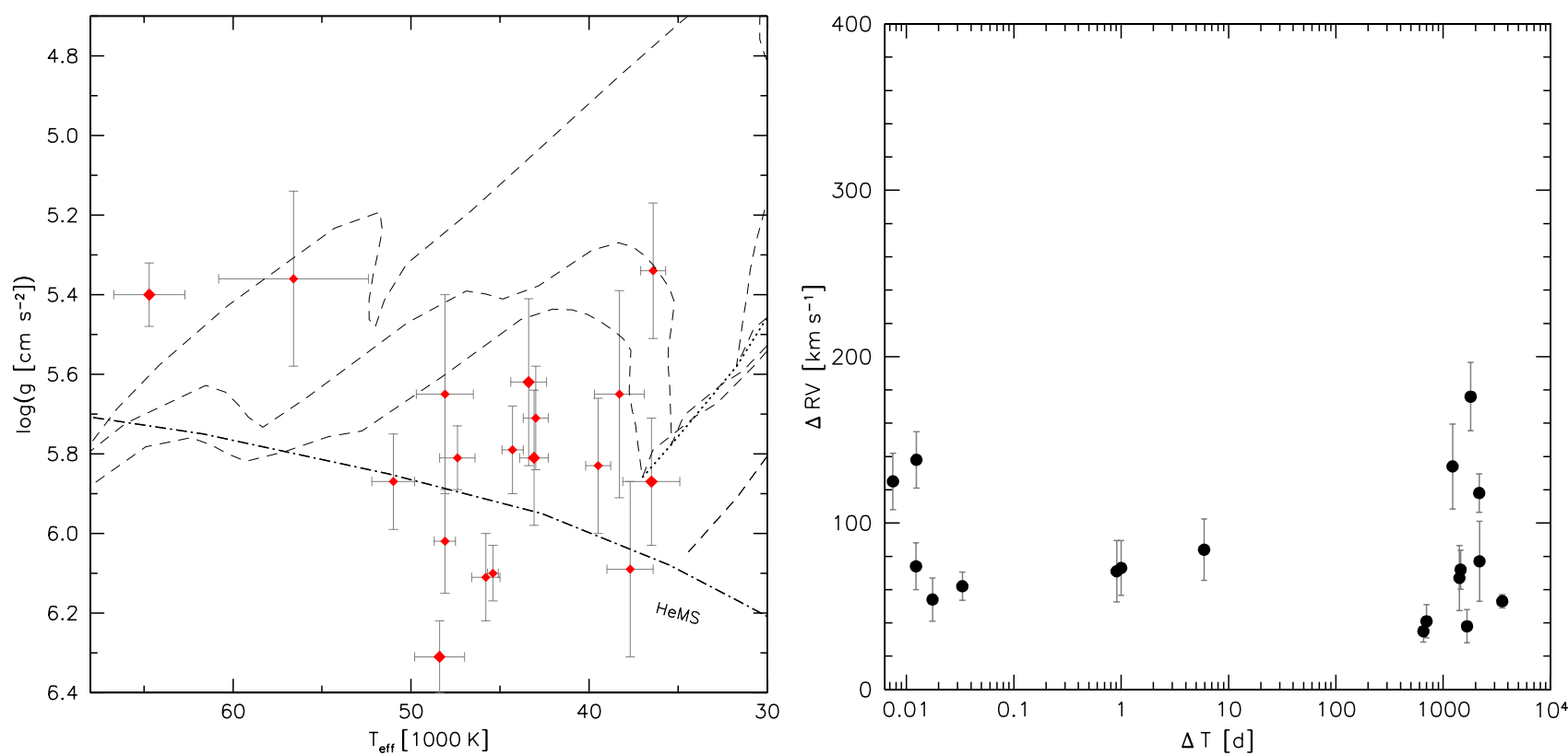

Fig. 4. Left panel: $T_{\text {eff }}-\log g$ diagram of $\mathrm{RV}$ variable helium-rich sdOB and sdO stars (see Fig. 1). The size of the symbols scales with $\Delta \mathrm{RV} \mathrm{max}_{\text {max }}$. The helium main sequence (HeMS) and the HB band are superimposed with HB evolutionary tracks (dashed lines) for subsolar metallicity $(\log z=-1.48)$ from Dorman et al. (1993). The three tracks correspond to helium core masses of $0.488,0.490$ and $0.495 M_{\odot}$ (from bottom-left to top-right). Right panel: highest RV shift between individual spectra plotted against time difference between the corresponding observing epochs for helium-rich sdO and sdOB stars (see Fig. 2). 
A\&A 602, C2 (2017)

Table 2. Parameters of 101 hydrogen-rich hot subdwarfs (65 RV variable, 36 RV variable candidates).

\begin{tabular}{|c|c|c|c|c|c|c|c|c|c|c|}
\hline Name & Class & $\begin{array}{l}m_{V} \\
{[\mathrm{mag}]}\end{array}$ & $\begin{array}{l}T_{\text {eff }} \\
{[\mathrm{K}]}\end{array}$ & $\log g$ & $\log y$ & $\begin{array}{l}d \\
{[\mathrm{kpc}]}\end{array}$ & $\begin{array}{l}\Delta t \\
{[\mathrm{~d}]}\end{array}$ & $\begin{array}{l}\Delta \mathrm{RV}_{\max } \\
{\left[\mathrm{km} \mathrm{s}^{-1}\right]}\end{array}$ & $N$ & $\ln p$ \\
\hline $\mathbf{J} 082332.09+113641.9^{b}$ & $\mathrm{sdB}$ & 16.7 & $31200 \pm 600$ & $5.79 \pm 0.06$ & $-2.0 \pm 0.1$ & $2.6_{-02}^{+0.2}$ & 53.9447 & $359.0 \pm 6.5$ & 22 & $<-680$ \\
\hline $\mathbf{J 1 1 3 8 4 0 . 6 8 - 0 0 3 5 3 1 . 7 ^ { c }}$ & $\mathrm{sdB}$ & 14.5 & $31200 \pm 600$ & $5.54 \pm 0.09$ & $<-3.0$ & $1.2_{-0 .}^{-0.2}$ & 3361.5592 & $332.0 \pm 14.0$ & 31 & $<-680$ \\
\hline $\mathbf{J} 165404.26+303701.8^{c}$ & $\mathrm{sdB}$ & 15.4 & $24900 \pm 800$ & $5.39 \pm 0.12$ & $-2.4 \pm 0.1$ & $1.8_{-0.3}^{+0.1}$ & 2.9365 & $271.0 \pm 17.0$ & 38 & $<-680$ \\
\hline $\mathbf{J} 225638.34+065651.1^{c}$ & $\mathrm{sdB}$ & 15.3 & $28500 \pm 500$ & $5.64 \pm 0.05$ & $-2.3 \pm 0.2$ & $1.5_{-0.1}^{+0.3}$ & 42.3494 & $269.0 \pm 14.0$ & 50 & $<-680$ \\
\hline $\mathrm{J} 172624.10+274419.3^{c}$ & $\mathrm{sdB}$ & 16.0 & $32600 \pm 500$ & $5.84 \pm 0.05$ & $-2.2 \pm 0.1$ & $1.9_{-0.1}^{+0.1}$ & 55.9741 & $263.0 \pm 12.0$ & 38 & $<-680$ \\
\hline $\mathrm{J} 150513.52+110836.6^{c}$ & $\mathrm{sdB}$ & 15.4 & $33200 \pm 500$ & $5.80 \pm 0.10$ & $-2.3 \pm 0.1$ & $1.5_{-0.2}^{+0.1}$ & 43.6564 & $222.0 \pm 8.0$ & 42 & $<-680$ \\
\hline $\mathbf{J} 134632.66+281722.7^{b}$ & $\mathrm{sdB}$ & 14.9 & $28800 \pm 600$ & $5.46 \pm 0.07$ & $-2.6 \pm 0.2$ & $1.6_{-0.1}^{-0.2}$ & 0.9988 & $191.0 \pm 7.0$ & 41 & $<-680$ \\
\hline J002323.99-002953.2 $2^{c}$ & $\mathrm{sdB}$ & 15.5 & $29200 \pm 500$ & $5.69 \pm 0.05$ & $-2.0 \pm 0.1$ & $1.6_{-0.1}^{-0.1}$ & 1.0413 & $168.0 \pm 4.0$ & 47 & $<-680$ \\
\hline $\mathbf{J} 083006.17+475150.4^{b}$ & $\mathrm{sdB}$ & 16.0 & $25300 \pm 600$ & $5.38 \pm 0.06$ & $<-3.0$ & $2.5_{-0.2}^{-0.1}$ & 4405.6747 & $164.0 \pm 9.0$ & 37 & $<-680$ \\
\hline $\mathbf{J} 095238.93+625818.9^{b}$ & $\mathrm{sdB}$ & 14.8 & $27700 \pm 600$ & $5.59 \pm 0.06$ & $-2.6 \pm 0.1$ & $1.2_{-0.1}^{+0.2}$ & 1183.7390 & $154.0 \pm 8.0$ & 34 & $<-680$ \\
\hline $\mathbf{J} 162256.66+473051.1^{d}$ & $\mathrm{sdB}$ & 16.2 & $29000 \pm 600$ & $5.65 \pm 0.06$ & $-1.9 \pm 0.1$ & $2.3_{-0.2}^{+0.1}$ & 1.9832 & $135.0 \pm 4.5$ & 34 & $<-680$ \\
\hline $\mathbf{J} 012022.94+395059.4^{e}$ & $\mathrm{sdB}$ & 15.4 & $28500 \pm 100$ & $5.42 \pm 0.01$ & $-3.0 \pm 0.1$ & $2.1_{-0.0}^{+0.2}$ & 1358.9782 & $129.0 \pm 6.5$ & 22 & $<-680$ \\
\hline $\mathrm{J} 173606.25+315842.7$ & $\mathrm{sdB}$ & 17.0 & $31300 \pm 300$ & $5.87 \pm 0.09$ & $-2.5 \pm 0.2$ & $2.8_{-0.3}^{+0.0}$ & 1567.7104 & $195.0 \pm 12.0$ & 12 & -537.49 \\
\hline $\mathbf{J} 032138.67+053840.0^{b}$ & $\mathrm{sdB}$ & 15.0 & $30700 \pm 500$ & $5.74 \pm 0.06$ & $-2.4 \pm 0.1$ & $1.3_{-0.1}^{-0.3}$ & 1699.1435 & $110.0 \pm 9.0$ & 46 & -536.46 \\
\hline J191908.76+371423.9 & $\mathrm{sdB}$ & 17.2 & $28300 \pm 400$ & $5.69 \pm 0.10$ & $-2.7 \pm 0.3$ & $3.4_{-0.4}^{-0.1}$ & 68.8608 & $237.0 \pm 12.0$ & 15 & -526.21 \\
\hline $\mathbf{J} 102151.64+301011.9^{a}$ & $\mathrm{sdB}$ & 18.3 & $30700 \pm 500$ & $5.71 \pm 0.06$ & $<-3.0$ & $5.8_{-0.5}^{+0.5}$ & 14.9363 & $277.0 \pm 37.0$ & 19 & -508.16 \\
\hline $\mathbf{J} 204613.40-045418.7^{c}$ & $\mathrm{sdB}$ & 16.2 & $31600 \pm 500$ & $5.54 \pm 0.08$ & $<-3.0$ & $2.8_{-0.3}^{+0.5}$ & 286.2265 & $259.0 \pm 16.0$ & 22 & -480.28 \\
\hline $\mathrm{J} 173806.51+451701.7$ & $\mathrm{sdB}$ & 17.4 & $30500 \pm 500$ & $5.40 \pm 0.08$ & $<-3.0$ & $5.5_{-06}^{+0.3}$ & 1.9536 & $233.0 \pm 8.5$ & 13 & -461.58 \\
\hline $\mathbf{J} 183249.04+630910.7^{b}$ & $\mathrm{sdB}$ & 15.8 & $26800 \pm 700$ & $5.29 \pm 0.09$ & $-2.6 \pm 0.1$ & $2.7_{-0.3}^{-0.6}$ & 1487.7733 & $141.0 \pm 8.0$ & 17 & -453.62 \\
\hline $\mathrm{J} 164326.04+330113.1^{a}$ & $\mathrm{sdB}$ & 16.3 & $27900 \pm 500$ & $5.62 \pm 0.07$ & $-2.3 \pm 0.2$ & $2.4_{-0.2}^{+0.3}$ & 2.8085 & $175.0 \pm 5.5$ & 10 & -452.26 \\
\hline $\mathbf{J} 011857.19-002546.5^{b}$ & $\mathrm{sdB}$ & 14.9 & $27900 \pm 600$ & $5.55 \pm 0.07$ & $<-3.0$ & $1.3_{-0.1}^{+0.2}$ & 265.2187 & $140.0 \pm 8.0$ & 43 & -386.44 \\
\hline $\mathrm{J} 192059.78+372220.0^{f}$ & $\mathrm{sdB}$ & 15.8 & $27600 \pm 600$ & $5.40 \pm 0.10$ & $-2.5 \pm 0.3$ & $2.4_{-0.3}^{+0.1}$ & 1.9589 & $123.0 \pm 4.5$ & 15 & -319.72 \\
\hline $\mathbf{J} 150829.02+494050.9^{b}$ & $\mathrm{sdB}$ & 17.7 & $29600 \pm 600$ & $5.73 \pm 0.07$ & $-2.3 \pm 0.1$ & $4.3_{-0.4}^{+0.3}$ & 2161.9292 & $209.0 \pm 15.5$ & 58 & -269.10 \\
\hline $\mathrm{J} 180940.41+234328.4$ & $\mathrm{sdB}$ & 16.5 & $28500 \pm 300$ & $5.44 \pm 0.06$ & $-2.9 \pm 0.2$ & $3.3_{-0.3}^{+0.4}$ & 2909.9029 & $342.0 \pm 29.5$ & 36 & -215.11 \\
\hline $\mathrm{J} 183349.79+652056.3$ & $\mathrm{sdB}$ & 17.4 & $27200 \pm 500$ & $5.56 \pm 0.12$ & $-2.6 \pm 0.1$ & $4.1_{-0.0}^{-0.3}$ & 68.8591 & $177.0 \pm 9.5$ & 16 & -190.20 \\
\hline $\mathbf{J} 095101.28+034757.0^{b}$ & $\mathrm{sdB}$ & 15.9 & $29800 \pm 300$ & $5.48 \pm 0.04$ & $-2.8 \pm 0.3$ & $2.5_{-0.1}^{+0.6}$ & 1.0425 & $183.0 \pm 13.0$ & 31 & -170.79 \\
\hline $\mathbf{J} 082053.53+000843.4^{g}$ & $\mathrm{sdB}$ & 15.2 & $26700 \pm 900$ & $5.48 \pm 0.10$ & $-2.0 \pm 0.1$ & $1.6_{-0.2}^{+0.1}$ & 388.9794 & $99.0 \pm 11.5$ & 24 & -153.08 \\
\hline J080738.96-083322.6 & $\mathrm{sdB}$ & 17.2 & $27600 \pm 600$ & $5.61 \pm 0.17$ & $-2.7 \pm 0.3$ & $3.6_{-07}^{+0.2}$ & 0.0736 & $298.0 \pm 19.0$ & 27 & -136.25 \\
\hline $\mathbf{J 1 5 2 2 2 2 . 1 5 - 0 1 3 0 1 8 . 3 ^ { b }}$ & $\mathrm{sdB}$ & 17.8 & $25200 \pm 700$ & $5.47 \pm 0.09$ & $<-3.0$ & $5.2_{-0.6}^{+0.7}$ & 3.0055 & $173.0 \pm 30.0$ & 26 & -126.07 \\
\hline $\mathrm{J} 155628.34+011335.0^{a}$ & $\mathrm{sdB}$ & 16.2 & $32700 \pm 600$ & $5.51 \pm 0.08$ & $-2.9 \pm 0.2$ & $3.1_{-0.3}^{+0.0}$ & 4412.8910 & $118.0 \pm 10.5$ & 15 & -121.64 \\
\hline $\mathbf{J 1 1 3 2 4 1 . 5 8 - 0 6 3 6 5 2 . 8 ^ { b }}$ & $\mathrm{sdO}$ & 16.2 & $46400 \pm 1000$ & $5.89 \pm 0.07$ & $-2.9 \pm 0.2$ & $2.4_{-0.2}^{+0.3}$ & 1517.8240 & $103.0 \pm 10.0$ & 32 & -108.89 \\
\hline $\mathrm{J} 222850.00+391917.4$ & $\mathrm{sdB}$ & 16.4 & $33500 \pm 900$ & $5.80 \pm 0.10$ & $-1.7 \pm 0.1$ & $2.4_{-0.3}^{+0.2}$ & 2051.8410 & $104.0 \pm 7.5$ & 40 & -85.63 \\
\hline $\mathrm{J} 173057.94+320737.0$ & $\mathrm{sdB}$ & 16.2 & $28200 \pm 700$ & $5.40 \pm 0.05$ & $-2.9 \pm 0.2$ & $3.0_{-0.2}^{+0.3}$ & 1.9680 & $94.0 \pm 8.0$ & 6 & -69.42 \\
\hline J083334.76-045759.4 & $\mathrm{sdB}$ & 18.2 & $20500 \pm 700$ & $5.52 \pm 0.10$ & $<-3.0$ & $5.0_{-07}^{+0.2}$ & 14.8908 & $161.0 \pm 8.5$ & 11 & -66.11 \\
\hline $\mathrm{J} 164853.26+121703.0$ & $\mathrm{sdB}$ & 18.5 & $30400 \pm 500$ & $5.38 \pm 0.11$ & $<-3.0$ & $9.3_{-12}^{+1.7}$ & 0.0684 & $135.0 \pm 13.0$ & 11 & -64.89 \\
\hline $\mathrm{J} 072245.27+305233.4$ & $\mathrm{sdB}$ & 18.0 & $25900 \pm 700$ & $5.61 \pm 0.16$ & $-2.6 \pm 0.2$ & $5.0_{-0.9}^{+1.2}$ & 1.0019 & $123.0 \pm 12.0$ & 7 & -62.09 \\
\hline J093059.63+025032.3 & $\mathrm{sdB}$ & 15.0 & $30000 \pm 600$ & $5.67 \pm 0.18$ & $-2.7 \pm 0.2$ & $1.3_{-0.3}^{+0.9}$ & 2986.7695 & $91.0 \pm 9.0$ & 10 & -49.22 \\
\hline $\mathrm{J} 203526.46+141948.4$ & $\mathrm{sdB}$ & 18.7 & $30200 \pm 600$ & $5.57 \pm 0.07$ & $-2.9 \pm 0.2$ & $8.3_{-0.8}^{-0.3}$ & 1.0235 & $163.0 \pm 25.5$ & 12 & -33.12 \\
\hline $\mathrm{J} 203843.97+141706.0$ & sdOB & 18.7 & $36800 \pm 1000$ & $5.89 \pm 0.20$ & $-2.4 \pm 0.3$ & $6.8_{-1.5}^{+1.8}$ & 0.9067 & $102.0 \pm 10.5$ & 12 & -32.22 \\
\hline $\mathrm{J} 095229.62+301553.6^{a}$ & sdOB & 18.5 & $35200 \pm 1200$ & $5.05 \pm 0.17$ & $<-3.0$ & $16.0_{-3.3}^{+3.5}$ & 1155.7612 & $198.0 \pm 27.5$ & 5 & -28.52 \\
\hline $\mathrm{J} 154531.01+563944.7$ & $\mathrm{sdB}$ & 17.0 & $26200 \pm 900$ & $5.13 \pm 0.14$ & $-2.0 \pm 0.2$ & $5.5_{-10}^{+1.2 .3}$ & 2527.7769 & $70.0 \pm 8.5$ & 9 & -27.76 \\
\hline J200959.27-115519.9 & $\mathrm{sdB}$ & 18.7 & $29700 \pm 700$ & $5.31 \pm 0.08$ & $<-3.0$ & $10.7_{-1.2}^{+1.3}$ & 1.9832 & $156.0 \pm 23.0$ & 8 & -27.48 \\
\hline $\mathrm{J} 005107.01+004232.5$ & sdOB & 15.9 & $38500 \pm 300$ & $5.83 \pm 0.07$ & $-1.0 \pm 0.1$ & $2.0_{-0.2}^{+0.2}$ & 2.0256 & $54.0 \pm 6.5$ & 7 & -24.96 \\
\hline $\mathrm{J} 104248.94+033355.3$ & $\mathrm{sdO}$ & 17.6 & $41200 \pm 3200$ & $4.83 \pm 0.15$ & $-2.1 \pm 0.4$ & $14.5_{-2.8}^{+3.2}$ & 2246.6948 & $49.0 \pm 5.0$ & 2 & -24.34 \\
\hline $\mathrm{J} 181141.86+241902.7$ & $\mathrm{sdB}$ & 18.7 & - & - & - & --2.8 & 0.9972 & $248.0 \pm 26.0$ & 7 & -23.56 \\
\hline J071424.12+401645.9 & $\mathrm{sdB}$ & 18.2 & $27700 \pm 700$ & $5.38 \pm 0.11$ & $-2.6 \pm 0.1$ & $7.6_{-11}^{+1.2}$ & 2.9312 & $152.0 \pm 24.0$ & 9 & -23.37 \\
\hline $\mathrm{J} 204300.90+002145.0^{a}$ & $\mathrm{sdO}$ & 17.9 & $40200 \pm 700$ & $6.15 \pm 0.13$ & $-1.3 \pm 0.4$ & $3.6_{-0 .}^{+0.1}$ & 18.8480 & $65.0 \pm 6.5$ & 9 & -22.54 \\
\hline $\mathrm{J} 191645.87+371224.5$ & $\mathrm{sdB}$ & 18.3 & $33200 \pm 1000$ & $5.84 \pm 0.17$ & $-2.7 \pm 0.2$ & $5.6_{-1 .}^{-1.3}$ & 3.0338 & $134.0 \pm 23.5$ & 19 & -22.15 \\
\hline $\mathrm{J} 094750.71+162731.8$ & $\mathrm{sdB}$ & 17.4 & $30000 \pm 700$ & $6.25 \pm 0.31$ & $-2.2 \pm 0.3$ & $2.1_{-07}^{+1.1}$ & 0.8902 & $130.0 \pm 13.5$ & 5 & -20.08 \\
\hline $\mathrm{J} 115358.81+353929.0^{a}$ & sdOB & 16.6 & $29400 \pm 500$ & $5.49 \pm 0.06$ & $-2.5 \pm 0.3$ & $3.3_{-0.3}^{+0.1}$ & 1151.6544 & $79.0 \pm 9.5$ & 5 & -19.15 \\
\hline $\mathrm{J} 175125.67+255003.5^{a}$ & $\mathrm{sdB}$ & 17.4 & $30600 \pm 500$ & $5.48 \pm 0.08$ & $<-3.8$ & $5.0_{-0.5}^{+0.3}$ & 1533.6229 & $72.0 \pm 10.0$ & 8 & -16.50 \\
\hline $\mathrm{J} 125702.30+435245.8^{a}$ & $\mathrm{sdB}$ & 18.2 & $28000 \pm 1100$ & $5.77 \pm 0.17$ & $<-3.0$ & $4.9_{-1.0}^{+1.3}$ & 0.0098 & $63.0 \pm 16.5$ & 3 & -16.32 \\
\hline $\mathrm{J} 165446.26+182224.6$ & $\mathrm{sdB}$ & 18.6 & $30100 \pm 500$ & $5.50 \pm 0.08$ & $-1.7 \pm 0.1$ & $8.5_{-0.9}^{+1.0}$ & 1396.0335 & $48.0 \pm 5.5$ & 3 & -15.27 \\
\hline $\mathrm{J} 120855.51+403716.1$ & $\mathrm{sdB}$ & 18.6 & $34100 \pm 900$ & $5.98 \pm 0.13$ & $-1.5 \pm 0.1$ & $5.4_{-0.0}^{+1.0}$ & 0.0260 & $171.0 \pm 20.0$ & 7 & -14.61 \\
\hline $\mathrm{J} 164122.32+334452.0$ & $\mathrm{sdB}$ & 15.5 & $28200 \pm 500$ & $5.49 \pm 0.11$ & $-2.5 \pm 0.3$ & $1.9_{-0.3}^{+0.9}$ & 2213.5393 & $77.0 \pm 8.0$ & 8 & -14.60 \\
\hline $\mathrm{J} 211421.39+100411.4$ & sdOB & 18.4 & $36100 \pm 900$ & $5.48 \pm 0.13$ & $-2.5 \pm 0.3$ & $9.2_{-1.4}^{+1.6}$ & 1427.1132 & $69.0 \pm 12.0$ & 7 & -14.02 \\
\hline $\mathrm{J} 170810.97+244341.6^{a}$ & sdOB & 18.5 & $35600 \pm 800$ & $5.58 \pm 0.14$ & $-0.8 \pm 0.1$ & $8.5_{-1.4}^{+1.6}$ & 0.0125 & $160.0 \pm 26.0$ & 3 & -13.73 \\
\hline $\mathrm{J} 153411.10+543345.2^{a}$ & sdOB & 16.9 & $34800 \pm 700$ & $5.64 \pm 0.09$ & $-2.6 \pm 0.3$ & $3.8_{-0.4}^{-1.4}$ & 0.0184 & $83.0 \pm 18.5$ & 8 & -12.52 \\
\hline $\mathrm{J} 224518.65+220746.5$ & $\mathrm{sdB}$ & 16.6 & $34000 \pm 800$ & $5.82 \pm 0.07$ & $-2.2 \pm 0.1$ & $2.6_{-0.3}^{-0.4}$ & 1080.8857 & $70.0 \pm 11.5$ & 9 & -12.28 \\
\hline $\mathrm{J} 120613.40+205523.1$ & sdOB & 18.4 & $35000 \pm 500$ & $5.35 \pm 0.07$ & $<-3.0$ & $10.5_{-0.9}^{+1.0}$ & 2.9112 & $91.0 \pm 23.5$ & 10 & -11.37 \\
\hline $\mathrm{J} 204247.51+001913.9^{h}$ & $\mathrm{sdB}$ & 19.6 & $34200 \pm 400$ & $5.89 \pm 0.08$ & $-1.3 \pm 0.1$ & $9.6_{-10}^{+1.1 .9}$ & 1393.1941 & $69.0 \pm 10.0$ & 3 & -10.83 \\
\hline $\mathrm{J} 151314.23+234248.8$ & $\mathrm{sdB}$ & 17.1 & $28700 \pm 300$ & $5.69 \pm 0.10$ & $-2.3 \pm 0.2$ & $3.3_{-0.4}^{+0.0}$ & 2.0006 & $58.0 \pm 8.5$ & 3 & -10.83 \\
\hline J082944.75+132302.5 & sdOB & 17.2 & $39700 \pm 600$ & $5.42 \pm 0.04$ & $<-3.0$ & $6.1_{-0.3}^{+0.3}$ & 24.9992 & $90.0 \pm 16.5$ & 5 & -10.40 \\
\hline
\end{tabular}

Notes. Solved binaries are marked in bold face and their orbital parameters can be found in Kupfer et al. (2015) and references therein. ${ }^{(a)}$ Atmospheric parameters taken from Geier et al. (2011a). ${ }^{(b)}$ Atmospheric parameters taken from Kupfer et al. (2015). ${ }^{(c)}$ Atmospheric parameters taken from Geier et al. (2011b). ${ }^{(d)}$ Atmospheric parameters taken from Schaffenroth et al. (2014). ${ }^{(e)}$ Atmospheric parameters taken from $\emptyset$ stensen et al. (2013). ${ }^{(f)}$ Atmospheric parameters taken from Schaffenroth et al. (in prep.). ${ }^{(g)}$ Atmospheric parameters taken from Geier et al. (2011c). ${ }^{(h)}$ Atmospheric parameters derived from a spectrum taken with ESO-VLT/FORS1. ${ }^{(i)}$ Atmospheric parameters derived from a spectrum taken with WHT/ISIS. 
S. Geier et al.: The catalogue of radial velocity variable hot subluminous stars from the MUCHFUSS project (Corrigendum)

Table 2. continued.

\begin{tabular}{|c|c|c|c|c|c|c|c|c|c|c|}
\hline Name & Class & $\begin{array}{l}m_{V} \\
{[\mathrm{mag}]}\end{array}$ & $\begin{array}{l}T_{\text {eff }} \\
{[\mathrm{K}]}\end{array}$ & $\log g$ & $\log y$ & $\begin{array}{l}d \\
{[\mathrm{kpc}]}\end{array}$ & $\begin{array}{l}\Delta t \\
{[\mathrm{~d}]}\end{array}$ & $\begin{array}{l}\Delta \mathrm{RV}_{\max } \\
{\left[\mathrm{km} \mathrm{s}^{-1}\right]}\end{array}$ & $N$ & $\ln p$ \\
\hline $\mathrm{J} 074534.16+372718.5^{a}$ & $\mathrm{sdB}$ & 17.9 & $37500 \pm 500$ & $5.90 \pm 0.09$ & $<-3.0$ & $4.6_{-0.5}^{+0.5}$ & 0.0363 & $64.0 \pm 17.0$ & 8 & -9.74 \\
\hline $\mathrm{J} 202313.83+131254.9^{a}$ & $\mathrm{sdB}$ & 17.2 & $29600 \pm 600$ & $5.64 \pm 0.14$ & $-2.1 \pm 0.1$ & $3.8_{-0.6}^{+0.0}$ & 1201.7981 & $123.0 \pm 19.0$ & 5 & -9.20 \\
\hline J162610.34+130401.6 & $\mathrm{sdB}$ & 19.4 & $33900 \pm 500$ & $5.63 \pm 0.10$ & $-1.0 \pm 0.1$ & $12.1_{-15}^{+1.7}$ & 780.7541 & $51.0 \pm 8.0$ & 3 & -9.16 \\
\hline $\mathrm{J} 030607.95+382335.7^{i}$ & $\mathrm{sdO}$ & 16.8 & $30100 \pm 300$ & $5.64 \pm 0.03$ & $-2.1 \pm 0.1$ & $3.2_{-0.1}^{+0.1}$ & 2210.7452 & $48.0 \pm 6.5$ & 8 & -8.85 \\
\hline J204451.08-062753.8 & $\mathrm{sdO}$ & 20.0 & $57100 \pm 5200$ & $5.61 \pm 0.15$ & $-2.2 \pm 0.4$ & $21.4_{-4.2}^{+5.1}$ & 1087.0571 & $62.0 \pm 10.5$ & 3 & -7.88 \\
\hline J091615.49+132833.1 & $\mathrm{sdB}$ & 17.5 & $30900 \pm 400$ & $5.48 \pm 0.05$ & $<-3.0$ & $5.4_{-0.4}^{+0.4}$ & 0.9512 & $55.0 \pm 11.5$ & 3 & -7.58 \\
\hline $\mathrm{J} 163413.09+163109.5$ & $\mathrm{sdB}$ & 18.3 & $34600 \pm 900$ & $4.73 \pm 0.12$ & $-2.0 \pm 0.5$ & $20.7_{-3.1}^{+3.5}$ & 1105.3751 & $21.0 \pm 5.5$ & 3 & -7.44 \\
\hline $\mathrm{J} 123220.09+260913.3$ & $\mathrm{sdB}$ & 18.1 & $33700 \pm 1100$ & $5.40 \pm 0.16$ & $-1.3 \pm 0.2$ & $8.5_{-1.7}^{+2.0}$ & 1.0302 & $134.0 \pm 27.0$ & 5 & -7.36 \\
\hline $\mathrm{J} 185129.02+182358.8$ & $\mathrm{sdB}$ & 16.8 & $27800 \pm 700$ & $5.38 \pm 0.10$ & $<-3.0$ & $3.9_{-0.5}^{-0.1}$ & 0.0808 & $105.0 \pm 18.0$ & 22 & -7.33 \\
\hline $\mathrm{J} 220048.67+123612.4^{h}$ & $\mathrm{sdO}$ & 18.6 & $64200 \pm 2600$ & $5.63 \pm 0.11$ & $-1.3 \pm 0.1$ & $11.4_{-16}^{-1.5}$ & 2437.2535 & $53.0 \pm 9.5$ & 3 & -7.04 \\
\hline $\mathrm{J} 153752.95+160201.8$ & $\mathrm{sdB}$ & 18.4 & $32300 \pm 500$ & $5.47 \pm 0.07$ & $<-3.0$ & $8.5_{-0.8}^{+0.6}$ & 0.0361 & $68.0 \pm 12.5$ & 3 & -7.03 \\
\hline $\mathrm{J} 183229.22+402418.4$ & $\mathrm{sdO}$ & 15.7 & $40100 \pm 600$ & $5.35 \pm 0.11$ & $-2.0 \pm 0.2$ & $3.3_{-0.4}^{+0.0}$ & 3.0098 & $50.0 \pm 11.5$ & 5 & -6.82 \\
\hline $\mathrm{J} 181126.83+233413.7$ & $\mathrm{sdB}$ & 18.4 & - & - & - & - & 1.0156 & $121.0 \pm 20.5$ & 7 & -6.47 \\
\hline $\mathrm{J} 204448.63+153638.8^{a}$ & $\mathrm{sdB}$ & 17.9 & $29600 \pm 600$ & $5.57 \pm 0.09$ & $-2.2 \pm 0.1$ & $5.7_{-0.7}^{+0.7}$ & 3.0489 & $101.0 \pm 17.5$ & 7 & -6.41 \\
\hline $\mathrm{J} 185414.11+175200.2$ & $\mathrm{sdOB}$ & 16.9 & $35200 \pm 700$ & $5.89 \pm 0.08$ & $-1.4 \pm 0.1$ & $2.9_{-0.3}^{+0.3}$ & 6.0874 & $81.0 \pm 22.0$ & 10 & -6.25 \\
\hline $\mathrm{J} 171629.92+575121.2^{a}$ & sdOB & 18.2 & $37500 \pm 800$ & $5.57 \pm 0.10$ & $<-0.7$ & $7.8_{-0.9}^{+1.3}$ & 3195.9096 & $67.0 \pm 15.5$ & 12 & -6.14 \\
\hline $\mathrm{J} 184434.74+412158.7$ & $\mathrm{sdB}$ & 17.3 & $27200 \pm 500$ & $5.57 \pm 0.12$ & $-2.6 \pm 0.1$ & $4.0_{-06}^{+0.7}$ & 2.9795 & $56.0 \pm 14.0$ & 5 & -5.72 \\
\hline J091136.73+124015.2 & $\mathrm{sdB}$ & 18.2 & - & - & - & - & 0.0173 & $75.0 \pm 16.5$ & 3 & -5.31 \\
\hline $\mathrm{J} 151337.80+195012.5$ & $\mathrm{sdB}$ & 18.9 & - & - & - & - & 0.0354 & $98.0 \pm 33.5$ & 4 & -5.16 \\
\hline $\mathrm{J} 172727.55+091215.5^{i}$ & $\mathrm{sdO}$ & 17.5 & $40100 \pm 1100$ & $5.36 \pm 0.09$ & $<-2.1$ & $7.4_{-08}^{+0.9}$ & 0.0141 & $55.0 \pm 10.5$ & 6 & -5.10 \\
\hline $\mathrm{J} 112242.69+613758.5^{a}$ & $\mathrm{sdB}$ & 15.4 & $29300 \pm 500$ & $5.69 \pm 0.10$ & $-2.3 \pm 0.3$ & $1.5_{-0.2}^{+0.8}$ & 0.0469 & $83.0 \pm 18.5$ & 6 & -5.08 \\
\hline $\mathrm{J} 161140.50+201857.0^{a}$ & $\mathrm{sdOB}$ & 18.5 & $36900 \pm 700$ & $5.89 \pm 0.13$ & $-1.2 \pm 0.1$ & $6.1_{-0.9}^{+1.1}$ & 0.9472 & $108.0 \pm 23.5$ & 5 & -4.77 \\
\hline $\mathrm{J} 065044.30+383133.7$ & sdOB & 17.3 & $34200 \pm 400$ & $5.76 \pm 0.07$ & $-2.9 \pm 0.2$ & $3.9_{-0.3}^{+0.9}$ & 0.0131 & $88.0 \pm 13.5$ & 14 & -4.63 \\
\hline $\mathrm{J} 170645.57+243208.6^{a}$ & $\mathrm{sdB}$ & 17.8 & $32000 \pm 500$ & $5.59 \pm 0.07$ & $<-4.0$ & $5.5_{-0.5}^{+0.6}$ & 0.0125 & $46.0 \pm 12.0$ & 3 & -4.41 \\
\hline J083359.65-043521.9 & sdOB & 18.3 & $36100 \pm 500$ & $5.92 \pm 0.11$ & $-1.9 \pm 0.2$ & $5.5_{-0.7}^{+0.3}$ & 14.9765 & $88.0 \pm 25.5$ & 11 & -4.39 \\
\hline $\mathrm{J} 140545.25+014419.0^{a}$ & $\mathrm{sdB}$ & 15.8 & $27300 \pm 800$ & $5.37 \pm 0.16$ & $-1.9 \pm 0.2$ & $2.5_{-0.5}^{+0.1}$ & 0.0263 & $25.0 \pm 8.0$ & 3 & -4.12 \\
\hline $\mathrm{J} 160534.96+062733.5$ & $\mathrm{sdB}$ & 19.3 & - & - & - & - & 1.0113 & $132.0 \pm 41.0$ & 8 & -3.97 \\
\hline $\mathrm{J} 221920.67+394603.5$ & $\mathrm{sdO}$ & 17.3 & $47000 \pm 3500$ & $5.73 \pm 0.16$ & $<-3.0$ & $4.7_{-0.9}^{+1.2}$ & 62.8679 & $66.0 \pm 12.5$ & 8 & -3.93 \\
\hline $\mathrm{J} 183840.52+400226.8$ & $\mathrm{sdB}$ & 17.8 & $29300 \pm 900$ & $5.52 \pm 0.13$ & $-1.6 \pm 0.2$ & $5.5_{-0.9}^{+1.9}$ & 2.9795 & $74.0 \pm 20.0$ & 5 & -3.89 \\
\hline $\mathrm{J} 115716.37+612410.7^{a}$ & $\mathrm{sdB}$ & 17.2 & $29900 \pm 500$ & $5.59 \pm 0.08$ & $-3.2 \pm 0.8$ & $4.0_{-0.4}^{+0.9}$ & 2250.6902 & $102.0 \pm 27.0$ & 7 & -3.63 \\
\hline $\mathrm{J} 113303.70+290223.0^{a}$ & $\mathrm{sdB} / \mathrm{DA}$ & 18.9 & - & - & - & - & 0.0158 & $95.0 \pm 30.0$ & 3 & -3.39 \\
\hline $\mathrm{J} 161817.65+120159.6^{a}$ & $\mathrm{sdB}$ & 18.0 & $32100 \pm 1000$ & $5.35 \pm 0.23$ & $<0.0$ & $8.1_{-2.1}^{+2.8}$ & 0.0427 & $105.0 \pm 28.0$ & 4 & -3.35 \\
\hline J205101.72+011259.7 & $\mathrm{sdB}+\mathrm{X}$ & 17.6 & - & - & - & - & 0.0141 & $91.0 \pm 31.5$ & 8 & -3.28 \\
\hline $\mathrm{J} 133638.81+111949.4^{a}$ & $\mathrm{sdB}$ & 17.3 & $27500 \pm 500$ & $5.49 \pm 0.08$ & $-2.7 \pm 0.2$ & $4.4_{-0.5}^{+0.5}$ & 0.0301 & $48.0 \pm 14.0$ & 3 & -3.25 \\
\hline J094044.07+004759. $6^{h}$ & $\mathrm{sdB}$ & 19.1 & $37000 \pm 800$ & $5.82 \pm 0.13$ & $-0.1 \pm 0.1$ & $8.8_{-1.3}^{+1.5}$ & 2982.7971 & $30.0 \pm 8.5$ & 2 & -3.24 \\
\hline $\mathrm{J} 210454.89+110645.5^{a}$ & sdOB & 17.3 & $37800 \pm 700$ & $5.63 \pm 0.10$ & $-2.4 \pm 0.2$ & $4.9_{-0.6}^{+0.3}$ & 2548.0064 & $139.0 \pm 27.5$ & 9 & -3.14 \\
\hline $\mathrm{J} 211651.96+003328.5^{a}$ & $\mathrm{sdB}$ & 18.0 & $27900 \pm 800$ & $5.78 \pm 0.15$ & $-3.9 \pm 0.7$ & $4.3_{-0.8}^{+0.9}$ & 0.0161 & $47.0 \pm 15.0$ & 3 & -3.08 \\
\hline $\mathrm{J} 091428.87+125023.8$ & $\mathrm{sdB}$ & 18.0 & $33600 \pm 600$ & $5.54 \pm 0.11$ & $<-3.0$ & $7.0_{-09}^{+1.1}$ & 0.0176 & $49.0 \pm 13.5$ & 3 & -3.07 \\
\hline
\end{tabular}


Table 3. Parameters of 18 helium-rich hot subdwarfs (6 RV variable, $12 \mathrm{RV}$ variable candidates).

\begin{tabular}{|c|c|c|c|c|c|c|c|c|c|c|}
\hline Name & Class & $\begin{array}{l}m_{V} \\
{[\mathrm{mag}]}\end{array}$ & $\begin{array}{l}T_{\text {eff }} \\
{[\mathrm{K}]}\end{array}$ & $\log g$ & $\log y$ & $\begin{array}{l}d \\
{[\mathrm{kpc}]}\end{array}$ & $\begin{array}{l}\Delta t \\
{[\mathrm{~d}]}\end{array}$ & $\begin{array}{l}\Delta \mathrm{RV}_{\max } \\
{\left[\mathrm{km} \mathrm{s}^{-1}\right]}\end{array}$ & $N$ & $\ln p$ \\
\hline $\mathrm{J} 232757.46+483755.2^{a}$ & $\mathrm{He}-\mathrm{sdO}$ & 15.8 & $64700 \pm 2000$ & $5.40 \pm 0.08$ & $>+2.0$ & $4.2_{-0.4}^{+0.5}$ & 1799.6136 & $176.0 \pm 20.5$ & 59 & -680.31 \\
\hline $\mathrm{J} 141549.05+111213.9^{a}$ & $\mathrm{He}-\mathrm{sdO}$ & 16.1 & $43100 \pm 800$ & $5.81 \pm 0.17$ & $>+2.0$ & $2.4_{-0.4}^{+0.4}$ & 0.0075 & $125.0 \pm 17.0$ & 35 & -86.42 \\
\hline $\mathrm{J} 103549.68+092551.9^{a}$ & He-sdO & 16.3 & $48100 \pm 600$ & $6.02 \pm 0.13$ & $>+2.0$ & $2.2_{-0.3}^{+0.4}$ & 3541.9636 & $53.0 \pm 4.0$ & 6 & -54.25 \\
\hline $\mathrm{J} 170045.09+391830.3$ & $\mathrm{He}$-sdOB & 18.2 & $36500 \pm 1600$ & $5.87 \pm 0.16$ & $+0.1 \pm 0.1$ & $5.5_{-1.0}^{+1.2}$ & 2160.0414 & $118.0 \pm 11.5$ & 10 & -44.76 \\
\hline $\mathrm{J} 161014.87+045046.6$ & $\mathrm{He}-\mathrm{sdO}$ & 17.3 & $48400 \pm 1400$ & $6.31 \pm 0.09$ & $>+2.0$ & $2.5_{-0.3}^{+0.3}$ & 0.0124 & $138.0 \pm 17.0$ & 14 & -31.77 \\
\hline $\mathrm{J} 110215.45+024034.1^{a}$ & $\mathrm{He}-\mathrm{sdO}$ & 17.5 & $56600 \pm 4200$ & $5.36 \pm 0.22$ & $>+2.0$ & $8.9_{-2.2}^{+3.0}$ & 0.0332 & $62.0 \pm 8.5$ & 3 & -10.91 \\
\hline $\mathrm{J} 174516.32+244348.3^{a}$ & $\mathrm{He}-\mathrm{sdO}$ & 17.7 & $43400 \pm 1000$ & $5.62 \pm 0.21$ & $>+2.0$ & $6.2_{-1.4}^{+1.8}$ & 1220.5806 & $134.0 \pm 25.5$ & 13 & -8.81 \\
\hline $\mathrm{J} 160304.07+165953.8^{b}$ & $\mathrm{He}-\mathrm{sdO}$ & 16.9 & $45400 \pm 300$ & $6.10 \pm 0.07$ & $>+2.0$ & $2.5_{-0.2}^{+0.4}$ & 0.9087 & $71.0 \pm 18.5$ & 5 & -8.11 \\
\hline $\mathrm{J} 094856.95+334151.0^{a}$ & $\mathrm{He}-\mathrm{sdO}$ & 17.7 & $51000 \pm 1200$ & $5.87 \pm 0.12$ & $+1.8 \pm 0.5$ & $5.1_{-0.7}^{-0.2}$ & 0.0123 & $74.0 \pm 14.0$ & 3 & -7.73 \\
\hline $\mathrm{J} 152136.25+162150.3$ & $\mathrm{He}-\mathrm{sdO}$ & 17.1 & $47400 \pm 1000$ & $5.81 \pm 0.08$ & $+1.6 \pm 0.4$ & $4.0_{-0.4}^{+0.4}$ & 2175.9687 & $77.0 \pm 24.0$ & 9 & -5.94 \\
\hline $\mathrm{J} 163416.08+221141.0$ & He-sdOB & 15.5 & $38300 \pm 1400$ & $5.65 \pm 0.26$ & $>+2.0$ & $2.0_{-0.6}^{+0.8}$ & 653.3309 & $35.0 \pm 6.5$ & 6 & -5.55 \\
\hline $\mathrm{J} 233914.00+134214.3$ & $\mathrm{He}$-sdO & 17.6 & $48100 \pm 1600$ & $5.65 \pm 0.25$ & $>+2.0$ & $6.0_{-1.6}^{+2.1}$ & 1451.6391 & $72.0 \pm 11.8$ & 12 & -5.11 \\
\hline $\mathrm{J} 173034.09+272139.8^{c}$ & $\mathrm{He}$-sdO & 18.9 & $39500 \pm 700$ & $5.83 \pm 0.17$ & $+0.1 \pm 0.1$ & $8.1_{-15}^{+1.0}$ & 698.7112 & $41.0 \pm 10.0$ & 2 & -5.00 \\
\hline $\mathrm{J} 170214.00+194255.1^{b}$ & $\mathrm{He}-\mathrm{sdO}$ & 15.8 & $44300 \pm 600$ & $5.79 \pm 0.11$ & $>+2.0$ & $2.1_{-0.3}^{+0.3}$ & 1665.2088 & $38.0 \pm 10.0$ & 5 & -3.76 \\
\hline J081329.81+383326.9 & $\mathrm{He}-\mathrm{sdO}$ & 17.5 & $45800 \pm 800$ & $6.11 \pm 0.11$ & $+1.8 \pm 0.4$ & $3.3_{-0.4}^{+0.5}$ & 0.0175 & $54.0 \pm 13.0$ & 6 & -3.35 \\
\hline $\mathrm{J} 204940.85+165003.6^{a}$ & $\mathrm{He}-\mathrm{sdO}$ & 17.9 & $43000 \pm 700$ & $5.71 \pm 0.13$ & $>+2.0$ & $6.2_{-0.9}^{+1.1}$ & 5.9325 & $84.0 \pm 18.5$ & 7 & -3.13 \\
\hline $\mathrm{J} 160623.21+363005.4$ & $\mathrm{He}$-sdOB & 18.5 & $36400 \pm 700$ & $5.34 \pm 0.17$ & $-0.5 \pm 0.1$ & $11.3_{-2.1}^{+2.6}$ & 1414.9811 & $67.0 \pm 19.5$ & 2 & -3.04 \\
\hline
\end{tabular}

Notes. ${ }^{(a)}$ Atmospheric parameters taken from Geier et al. (2011a). ${ }^{(b)}$ Atmospheric parameters derived from a spectrum taken with ESOVLT/FORS1. ${ }^{(c)}$ Atmospheric parameters derived from a spectrum taken with WHT/ISIS.

Table 4. Parameters of 10 other types of hot stars (5 RV variable, $5 \mathrm{RV}$ variable candidates).

\begin{tabular}{|c|c|c|c|c|c|c|c|c|c|c|}
\hline Name & Class & $\begin{array}{l}m_{V} \\
{[\mathrm{mag}]}\end{array}$ & $\begin{array}{l}T_{\text {eff }} \\
{[\mathrm{K}]}\end{array}$ & $\log g$ & $\log y$ & $\begin{array}{l}d \\
{[\mathrm{kpc}]}\end{array}$ & $\begin{array}{l}\Delta t \\
{[\mathrm{~d}]}\end{array}$ & $\begin{array}{l}\Delta \mathrm{RV}_{\max } \\
{\left[\mathrm{km} \mathrm{s}^{-1}\right]}\end{array}$ & $N$ & $\ln p$ \\
\hline J131916.15-011404.9 & BHB & 16.4 & $17400 \pm 800$ & $4.55 \pm 0.15$ & $-1.9 \pm 0.2$ & $5.9_{-1.1}^{+1.4}$ & 2888.0925 & $46.0 \pm 9.0$ & 8 & -42.10 \\
\hline $\mathrm{J} 164121.22+363542.7$ & ВHB & 17.4 & $19300 \pm 1000$ & $4.55 \pm 0.10$ & $-1.9 \pm 0.2$ & $9.9_{-1.4}^{+1.7}$ & 1035.9093 & $99.0 \pm 9.0$ & 8 & -39.13 \\
\hline $\mathrm{J} 075732.18+184329.3^{a}$ & $\mathrm{O}(\mathrm{He})$ & 18.6 & $80000 \pm 2000$ & $5.00 \pm 0.30$ & $>+2.0$ & $29.6_{-9.0}^{+12.7}$ & 0.0216 & $107.0 \pm 22.0$ & 6 & -30.13 \\
\hline $\mathrm{J} 155610.40+254640.3^{b}$ & PG 1159 & 17.9 & $100000_{-10000}^{+15000}$ & $5.3 \pm 0.3$ & $>+2.0$ & $16.9_{-5.6}^{+8.9}$ & 231.1694 & $116.0 \pm 21.0$ & 10 & -17.98 \\
\hline J201302.58-105826.1 & MS-B & 18.5 & $16400 \pm 1400$ & $4.30 \pm 0.27$ & $-1.3 \pm 0.2$ & $51.8_{-16.4}^{+23.6}$ & 2.0155 & $61.0 \pm 11.5$ & 8 & -13.42 \\
\hline J093521.39+482432.4 & $\mathrm{O}(\mathrm{H})$ & 18.5 & $87700 \pm 20000$ & $5.68 \pm 0.16$ & $-1.0 \pm 0.3$ & $12.0_{-3.3}^{+3.7}$ & 2269.7542 & $38.0 \pm 7.5$ & 2 & -6.97 \\
\hline $\mathrm{J} 161253.21+060538.7$ & MS-B & 15.5 & $15700 \pm 1400$ & $4.18 \pm 0.29$ & $-1.0 \pm 0.2$ & $14.4_{-4.8}^{+7.3}$ & 811.5968 & $38.0 \pm 7.0$ & 10 & -6.84 \\
\hline $\mathrm{J} 020531.40+134739.8^{c}$ & BHB & 18.4 & $17400 \pm 700$ & $4.26 \pm 0.13$ & $-1.7 \pm 0.2$ & $20.3_{-3.4}^{+4.0}$ & 2781.1087 & $28.0 \pm 7.0$ & 3 & -3.64 \\
\hline $\mathrm{J} 144023.58+135454.7$ & ВHB & 18.3 & $18900 \pm 700$ & $4.50 \pm 0.15$ & $-1.9 \pm 0.3$ & $16.1_{-3.0}^{+3.6}$ & 0.0528 & $78.0 \pm 24.0$ & 4 & -3.15 \\
\hline $\mathrm{J} 171947.87+591604.2$ & MS-B & 16.9 & $15100 \pm 600$ & $4.10 \pm 0.19$ & $-0.9 \pm 0.2$ & $29.2_{-6.5}^{+8.3}$ & 2568.7218 & $32.0 \pm 6.5$ & 10 & -3.11 \\
\hline
\end{tabular}

Notes. ${ }^{(a)}$ Atmospheric parameters taken from Werner et al. (2014). ${ }^{(b)}$ Atmospheric parameters taken from Reindl et al. (2016). ${ }^{(c)}$ Atmospheric parameters derived from a spectrum taken with ESO-VLT/FORS1. 
Table 5. Parameters of 67 stars with non-significant RV variations.

\begin{tabular}{|c|c|c|c|c|c|c|c|c|c|c|}
\hline Name & Class & $\begin{array}{l}m_{V} \\
{[\mathrm{mag}]}\end{array}$ & $\begin{array}{l}T_{\text {eff }} \\
{[\mathrm{K}]}\end{array}$ & $\log g$ & $\log y$ & $\begin{array}{l}d \\
{[\mathrm{kpc}]}\end{array}$ & $\begin{array}{l}\Delta t \\
\text { [d] }\end{array}$ & $\begin{array}{l}\Delta \mathrm{RV}_{\max } \\
{\left[\mathrm{km} \mathrm{s}^{-1}\right]}\end{array}$ & $N$ & $\ln p$ \\
\hline J112014.86+412127.3 & $\mathrm{sdB}$ & 18.1 & - & - & - & - & 1503.8023 & $23.0 \pm 7.5$ & 2 & -2.98 \\
\hline J173614.19+335249.5 & $\mathrm{sdB}$ & 18.8 & - & - & - & - & 0.0410 & $85.0 \pm 26.0$ & 5 & -2.97 \\
\hline J092520.70+470330. $6^{a}$ & $\mathrm{sdB}$ & 17.7 & $28100 \pm 900$ & $5.17 \pm 0.15$ & $-2.5 \pm 0.2$ & $7.5_{-14}^{+1.7}$ & 0.0126 & $40.0 \pm 12.5$ & 3 & -2.88 \\
\hline $\mathrm{J} 171617.33+553446.7^{a}$ & $\mathrm{sdB}$ & 17.2 & $32900 \pm 900$ & $5.48 \pm 0.09$ & $<-3.0$ & $4.9_{-06}^{+0.7}$ & 0.0125 & $130.0 \pm 40.5$ & 9 & -2.85 \\
\hline J064809.54+380850.1 & $\mathrm{sdB}$ & 18.4 & $29300 \pm 800$ & $5.26 \pm 0.13$ & $-2.8 \pm 0.3$ & $9.8_{-16}^{+1.9}$ & 0.9989 & $48.0 \pm 13.0$ & 5 & -2.85 \\
\hline $\mathrm{J} 075937.15+541022.2^{a}$ & $\mathrm{sdB}$ & 17.8 & $31300 \pm 700$ & $5.30 \pm 0.10$ & $-3.3 \pm 0.3$ & $7.6_{-1.0}^{+1.1}$ & 0.0233 & $40.0 \pm 18.5$ & 3 & -2.75 \\
\hline J001844.33-093855.0 & $\mathrm{sdB}$ & 18.8 & - & - & - & - & 1169.8455 & $27.0 \pm 8.0$ & 3 & -2.75 \\
\hline $\mathrm{J} 112414.45+402637.1^{a}$ & He-sdO & 18.0 & $47100 \pm 1000$ & $5.81 \pm 0.23$ & $>+1.7$ & $5.9_{-14}^{+1.9}$ & 0.0215 & $62.0 \pm 18.5$ & 3 & -2.65 \\
\hline $\mathrm{J} 161059.80+053625.2^{b}$ & $\mathrm{He}-\mathrm{sdO}$ & 17.2 & $46300 \pm 700$ & $6.22 \pm 0.10$ & $+1.0 \pm 0.6$ & $2.6_{-0.3}^{+0.3}$ & 751.7674 & $38.0 \pm 9.5$ & 4 & -2.64 \\
\hline $\mathrm{J} 130439.57+312904.8^{a}$ & $\mathrm{sdOB}$ & 17.1 & $38100 \pm 600$ & $5.69 \pm 0.12$ & $-0.4 \pm 0.1$ & $4.1_{-06}^{+0.6}$ & 0.0163 & $49.0 \pm 27.5$ & 3 & -2.63 \\
\hline $\mathrm{J} 143347.59+075416.9$ & $\mathrm{sdOB}$ & 16.7 & $36600 \pm 600$ & $6.16 \pm 0.13$ & $<-0.5$ & $1.9_{-03}^{+0.3}$ & 805.7659 & $52.0 \pm 10.5$ & 11 & -2.61 \\
\hline $\mathrm{J} 151415.66-012925.2^{a}$ & $\mathrm{He}$-sdO & 17.0 & $48200 \pm 500$ & $5.85 \pm 0.08$ & $+1.7 \pm 0.4$ & $3.6_{-03}^{+0.4}$ & 3.9687 & $66.0 \pm 20.5$ & 5 & -2.58 \\
\hline $\mathrm{J} 153540.30+173458.8$ & $\mathrm{sdB}$ & 18.0 & - & - & - & - & 0.0168 & $58.0 \pm 16.5$ & 3 & -2.57 \\
\hline $\mathrm{J} 202758.63+773924.5^{a}$ & $\mathrm{sdO}$ & 17.9 & $46200 \pm 3200$ & $5.48 \pm 0.18$ & $-2.8 \pm 0.9$ & $8.2_{-18}^{+2.2}$ & 1.9601 & $114.0 \pm 33.0$ & 3 & -2.48 \\
\hline $\mathrm{J} 215648.71+003620.7^{a}$ & $\mathrm{sdB}$ & 18.0 & $30800 \pm 800$ & $5.77 \pm 0.12$ & $-2.2 \pm 0.3$ & $4.7_{-07}^{+0.8}$ & 822.1114 & $100.0 \pm 28.0$ & 6 & -2.38 \\
\hline J073701.45+225637.6 & $\mathrm{sdB}$ & 16.8 & $28100 \pm 300$ & $5.45 \pm 0.04$ & $<-3.0$ & $3.7_{-0.2}^{+0.2}$ & 2.0639 & $53.0 \pm 14.5$ & 5 & -2.36 \\
\hline J220810.05+115913.9 & $\mathrm{sdB}$ & 17.4 & $27200 \pm 600$ & $5.23 \pm 0.07$ & $-2.3 \pm 0.3$ & $6.1_{-06}^{-0.6}$ & 2172.7020 & $42.0 \pm 12.5$ & 5 & -2.31 \\
\hline J172919.04+072204.5 & $\mathrm{sdO}$ & 17.3 & $49200 \pm 1900$ & $5.78 \pm 0.12$ & $-3.0 \pm 0.4$ & $4.6_{-07}^{+0.8}$ & 0.0179 & $58.0 \pm 20.0$ & 5 & -2.22 \\
\hline J100019.98-003413.3 & $\mathrm{O}(\mathrm{H})$ & 17.8 & $93700 \pm 10700$ & $5.88 \pm 0.10$ & $-0.6 \pm 0.2$ & $7.3_{-1.1}^{+1.3}$ & 3.0114 & $135.0 \pm 28.0$ & 16 & -2.20 \\
\hline $\mathrm{J} 031226.01+001018.2$ & $\mathrm{sdB}$ & 19.2 & - & - & - & - & 2552.8670 & $71.0 \pm 30.5$ & 2 & -2.17 \\
\hline $\mathrm{J} 204546.81-054355.6^{a}$ & $\mathrm{sdB}$ & 17.9 & $35500 \pm 500$ & $5.47 \pm 0.09$ & $-1.4 \pm 0.2$ & $7.3_{-08}^{+0.9}$ & 0.0128 & $41.0 \pm 16.5$ & 4 & -2.15 \\
\hline $\mathrm{J} 133200.95+673325.7$ & sdOB & 17.2 & $37400 \pm 500$ & $5.90 \pm 0.09$ & $-1.5 \pm 0.1$ & $3.4_{-0.4}^{+0.4}$ & 2584.9083 & $53.0 \pm 14.5$ & 7 & -2.09 \\
\hline J120427.94+172745.3 & $\mathrm{sdB}$ & 18.3 & $25100 \pm 900$ & $5.25 \pm 0.15$ & $-2.6 \pm 0.4$ & $8.2_{-15}^{+1.9}$ & 0.0282 & $68.0 \pm 29.0$ & 3 & -2.05 \\
\hline $\mathrm{J} 204550.97+153536.3$ & $\mathrm{sdB}$ & 18.2 & $30300 \pm 500$ & $5.62 \pm 0.09$ & $<-3.0$ & $6.3_{-0.8}^{+0.8}$ & 5.9148 & $58.0 \pm 13.5$ & 7 & -1.98 \\
\hline $\mathrm{J} 135807.96+261215.5^{a}$ & $\mathrm{sdB}$ & 17.9 & $33500 \pm 600$ & $5.66 \pm 0.10$ & $>+2.0$ & $5.8_{-0.7}^{+0.8}$ & 0.0302 & $86.0 \pm 26.0$ & 6 & -1.89 \\
\hline $\mathrm{J} 113935.45+614953.9^{a}$ & $\mathrm{sdB}$ & 16.9 & $28800 \pm 900$ & $5.27 \pm 0.15$ & $-2.8 \pm 0.3$ & $4.9_{-09}^{+1.11}$ & 0.0112 & $30.0 \pm 10.5$ & 3 & -1.86 \\
\hline J161938.64+252122.4 & He-sdOB & 17.5 & $35000 \pm 2000$ & $5.80 \pm 0.33$ & $-0.4 \pm 0.2$ & $4.3_{-15}^{+2.1}$ & 0.9716 & $67.0 \pm 26.0$ & 3 & -1.81 \\
\hline $\mathrm{J} 155343.39+131330.4$ & $\mathrm{sdOB}$ & 18.5 & $36300 \pm 500$ & $5.63 \pm 0.16$ & $-0.8 \pm 0.1$ & $8.1_{-14}^{+1.7}$ & 0.0160 & $64.0 \pm 24.0$ & 3 & -1.77 \\
\hline $\mathrm{J} 110256.32+010012.3^{b}$ & BHB & 18.5 & $17300 \pm 800$ & $4.32 \pm 0.14$ & $-2.1 \pm 0.2$ & $19.5_{-35}^{+14.3}$ & 2735.5338 & $24.0 \pm 9.0$ & 3 & -1.77 \\
\hline J160450.44+051909.2 & He-sdOB & 18.5 & $38100 \pm 700$ & $5.22 \pm 0.27$ & $+1.2 \pm 0.2$ & $13.7_{-38}^{+5.2}$ & 0.9736 & $145.0 \pm 61.0$ & 8 & -1.75 \\
\hline J082657.29+122818.1 & $\mathrm{sdOB}$ & 17.1 & $36500 \pm 400$ & $5.83 \pm 0.12$ & $-1.4 \pm 0.1$ & $3.4_{-05}^{+0.5}$ & 0.0142 & $67.0 \pm 22.0$ & 4 & -1.73 \\
\hline $\mathrm{J} 152705.03+110843.9^{a}$ & $\mathrm{sdOB}$ & 17.3 & $37600 \pm 500$ & $5.62 \pm 0.10$ & $-0.5 \pm 0.1$ & $4.8_{-0.6}^{+0.6}$ & 0.0543 & $43.0 \pm 12.0$ & 5 & -1.73 \\
\hline J052544.93+630726. $0^{a}$ & $\mathrm{sdOB}$ & 17.7 & $35600 \pm 800$ & $5.85 \pm 0.10$ & $-1.6 \pm 0.2$ & $4.3_{-0}^{+0.6}$ & 0.0264 & $42.0 \pm 15.0$ & 5 & -1.73 \\
\hline $\mathrm{J} 100535.76+223952.1^{a}$ & $\mathrm{sdB}$ & 18.4 & $29000 \pm 700$ & $5.43 \pm 0.13$ & $-2.7 \pm 0.2$ & $7.9_{-13}^{+1.5}$ & 0.0192 & $41.0 \pm 14.0$ & 4 & -1.71 \\
\hline $\mathrm{J} 204149.38+003555.8^{b}$ & BHB & 19.0 & $19400 \pm 2200$ & $4.02 \pm 0.29$ & $-2.1 \pm 0.4$ & $38.3_{-1.3}^{+20.3}$ & 38.0700 & $26.0 \pm 10.5$ & 3 & -1.71 \\
\hline $\mathrm{J} 164204.37+440303.2$ & $\mathrm{sdB}$ & 16.8 & $29300 \pm 800$ & $5.09 \pm 0.13$ & $-2.5 \pm 0.3$ & $5.7_{-0.9}^{+1.1 .4}$ & 0.0273 & $31.0 \pm 11.5$ & 4 & -1.68 \\
\hline J090252.99+073533.9 & He-sdO & 17.4 & $40100 \pm 500$ & $5.91 \pm 0.19$ & $>+2.0$ & $\begin{array}{l}-1.9 \\
3.7_{-0}^{+0.9}\end{array}$ & 1612.4334 & $67.0 \pm 27.0$ & 5 & -1.65 \\
\hline J090957.82+622927.0 & sdO & 16.4 & $48000 \pm 4900$ & $5.68 \pm 0.17$ & $-1.7 \pm 0.6$ & $3.4_{-0.8}^{+1.0}$ & 0.0461 & $37.0 \pm 12.0$ & 4 & -1.64 \\
\hline $\mathrm{J} 152458.81+181940.5$ & $\mathrm{sdO}$ & 18.3 & $52300 \pm 2500$ & $5.28 \pm 0.08$ & $-2.8 \pm 0.3$ & $13.5_{-1.5}^{+1.7}$ & 0.0155 & $41.0 \pm 15.0$ & 3 & -1.60 \\
\hline $\mathrm{J} 112140.20+183613.7$ & $\mathrm{sdB}$ & 18.6 & $28100 \pm 500$ & $5.46 \pm 0.10$ & $-1.8 \pm 0.1$ & $8.3_{-10}^{+1.2}$ & 0.9796 & $71.0 \pm 26.0$ & 4 & -1.57 \\
\hline $\mathrm{J} 151254.55+150447.0$ & $\mathrm{sdOB}$ & 17.8 & $38300 \pm 600$ & $6.01 \pm 0.10$ & $-1.5 \pm 0.2$ & $4.0_{-0.5}^{+0.5}$ & 0.0229 & $65.0 \pm 28.0$ & 3 & -1.54 \\
\hline $\mathrm{J} 233406.11+462249.3^{a}$ & $\mathrm{sdB}$ & 17.7 & $34600 \pm 500$ & $5.71 \pm 0.09$ & $-1.3 \pm 0.1$ & $4.9_{-06}^{+0.6}$ & 0.0248 & $31.0 \pm 12.0$ & 3 & -1.53 \\
\hline J095054.97+460405.2 & $\mathrm{sdB}$ & 18.0 & $28500 \pm 500$ & $5.24 \pm 0.07$ & $-2.3 \pm 0.3$ & $8.1_{-0.8}^{+0.8}$ & 0.0390 & $42.0 \pm 16.5$ & 3 & -1.52 \\
\hline J081304.04-071306.5 & $\mathrm{He}$-sdO & 18.6 & $48200 \pm 900$ & $5.93 \pm 0.14$ & $+1.8 \pm 0.5$ & $7.0_{-1.1}^{+1.1}$ & 0.9897 & $137.0 \pm 41.0$ & 7 & -1.50 \\
\hline J112526.95+112902.6 & $\mathrm{sdOB}$ & 17.4 & $36100 \pm 700$ & $6.06 \pm 0.12$ & $-0.8 \pm 0.1$ & $2.9_{-04}^{-1.5}$ & 0.0142 & $70.0 \pm 31.0$ & 4 & -1.50 \\
\hline $\mathrm{J} 163834.68+265110.2$ & $\mathrm{sdOB}$ & 17.0 & $36000 \pm 300$ & $5.80 \pm 0.05$ & $-1.6 \pm 0.1$ & $3.4^{-0.4}+0.2$ & 0.0159 & $40.0 \pm 13.0$ & 4 & -1.50 \\
\hline J203017.81+131849.2 & $\mathrm{sdOB}$ & 16.8 & $37100 \pm 500$ & $5.92 \pm 0.09$ & $-1.4 \pm 0.1$ & $2.7_{-3.3}^{+0.3}$ & 1200.7860 & $52.0 \pm 20.0$ & 5 & -1.47 \\
\hline $\mathrm{J} 130059.20+005711.7^{a}$ & $\mathrm{sdOB}$ & 16.5 & $40700 \pm 500$ & $5.53 \pm 0.10$ & $-0.6 \pm 0.1$ & $3.9^{-0.5}$ & 0.0123 & $36.0 \pm 14.5$ & 3 & -1.43 \\
\hline $\mathrm{J} 085727.65+424215.4^{a}$ & $\mathrm{He}$-sdO & 18.5 & $39500 \pm 1900$ & $5.63 \pm 0.24$ & $+0.2 \pm 0.2$ & $8.7_{-2.2}^{+3.0}$ & 0.0657 & $111.0 \pm 39.5$ & 4 & -1.26 \\
\hline J074551.13+170600.3 & $\mathrm{sdOB}$ & 17.1 & $35600 \pm 400$ & $5.54 \pm 0.05$ & $-2.8 \pm 0.1$ & $4.7_{-03}^{+0.2}$ & 9.9390 & $65.0 \pm 12.0$ & 18 & -1.26 \\
\hline $\mathrm{J} 110445.01+092530.9^{a}$ & $\mathrm{sdOB}$ & 16.3 & $35900 \pm 800$ & $5.41 \pm 0.07$ & $-2.1 \pm 0.4$ & $3.8_{-0.4}^{+0.3}$ & 0.0396 & $34.0 \pm 12.0$ & 4 & -1.25 \\
\hline $\mathrm{J} 012739.35+404357.8^{a}$ & $\mathrm{sdO}$ & 16.8 & $48300 \pm 3200$ & $5.67 \pm 0.10$ & $-1.3 \pm 0.2$ & $4.1_{-06}^{+0.7}$ & 0.0369 & $45.0 \pm 17.0$ & 8 & -1.23 \\
\hline J172816.87+074839.0 & $\mathrm{sdB}$ & 18.4 & $30700 \pm 700$ & $5.37 \pm 0.09$ & $-2.5 \pm 0.4$ & $9.0_{-1.2}^{+1.2}$ & 1.9962 & $75.0 \pm 34.0$ & 7 & -1.11 \\
\hline $\mathrm{J} 143153.05-002824.3^{a}$ & $\mathrm{sdOB}$ & 18.1 & $37300 \pm 800$ & $6.02 \pm 0.16$ & $-0.8 \pm 0.1$ & $4.4_{-0.9}^{+0.9}$ & 0.0120 & $64.0 \pm 20.5$ & 8 & -1.05 \\
\hline $\mathrm{J} 225150.80-082612.7^{b}$ & BHB & 18.4 & $19000 \pm 500$ & $4.98 \pm 0.09$ & $-1.8 \pm 0.3$ & $9.5_{-1}^{+1}$ & 2411.2964 & $20.0 \pm 7.0$ & 5 & -1.04 \\
\hline J074806.15+342927.7 & $\mathrm{sdOB}$ & 17.3 & $35100 \pm 800$ & $5.72 \pm 0.08$ & $-1.7 \pm 0.1$ & $4.3_{-0}^{+0.5}$ & 5.9453 & $42.0 \pm 12.5$ & 12 & -0.95 \\
\hline $\mathrm{J} 111225.70+392332.7$ & sdOB & 17.6 & $37800 \pm 500$ & $5.76 \pm 0.11$ & $-0.6 \pm 0.1$ & $4.9_{-0.6}^{+0.7}$ & 0.0563 & $104.0 \pm 28.0$ & 13 & -0.92 \\
\hline $\mathrm{J} 134352.14+394008.3^{a}$ & He-sdOB & 18.2 & $36000 \pm 2100$ & $4.78 \pm 0.30$ & $-0.2 \pm 0.2$ & $18.8_{-61}^{+8.5}$ & 0.0224 & $53.0 \pm 27.0$ & 3 & -0.89 \\
\hline $\mathrm{J} 163702.78-011351.7^{a}$ & $\mathrm{He}$-sdO & 17.3 & $46100 \pm 700$ & $5.92 \pm 0.22$ & $>+2.0$ & $3.8_{-09}^{+1.1}$ & 0.0853 & $100.0 \pm 42.5$ & 12 & -0.85 \\
\hline J174442.35+263829.9 & $\mathrm{sdOB}$ & 17.9 & - & - & - & - & 0.0384 & $88.0 \pm 44.0$ & 7 & -0.84 \\
\hline $\mathrm{J} 180757.08+230133.0$ & He-sdO & 17.1 & $42700 \pm 1000$ & $6.04 \pm 0.21$ & $>+2.0$ & $2.9_{-0.7}^{+0.8}$ & 0.9992 & $39.0 \pm 19.0$ & 4 & -0.83 \\
\hline J204623.12-065926.8 & $\mathrm{O}(\mathrm{H})$ & 17.7 & $79500 \pm 12500$ & $5.74 \pm 0.13$ & $-1.1 \pm 0.2$ & $\begin{array}{l}7.6_{-16}^{+1.9} \\
7.9\end{array}$ & 1376.1081 & $47.0 \pm 18.0$ & 5 & -0.64 \\
\hline J075818.49+102742.5 & $\mathrm{sdOB}$ & 16.4 & $37400 \pm 600$ & $5.51 \pm 0.05$ & $<-3.0$ & $3.6_{-02}^{+0.2}$ & 0.0596 & $32.0 \pm 12.5$ & 6 & -0.57 \\
\hline J215053.84+131650.5 & $\mathrm{sdB}+\mathrm{X}$ & 17.0 & - & - & - & - & 0.0154 & $24.0 \pm 13.5$ & 4 & -0.56 \\
\hline J215307.34-071948.3 & $\mathrm{sdB}$ & 17.1 & $33100 \pm 1300$ & $5.74 \pm 0.15$ & $-2.0 \pm 0.2$ & $3.6_{-0.7}^{+0.8}$ & 24.9831 & $50.0 \pm 27.5$ & 13 & -0.42 \\
\hline $\mathrm{J} 113418.00+015322.1^{a}$ & $\mathrm{sdB}$ & 17.7 & $29700 \pm 1200$ & $4.83 \pm 0.16$ & $<-4.0$ & $11.8_{-24}^{+2.9}$ & 0.0757 & $46.0 \pm 20.0$ & 6 & -0.42 \\
\hline J170716.53+275410.4 & $\mathrm{sdB}$ & 16.7 & $30200 \pm 1400$ & $5.62 \pm 0.16$ & $<-3.0$ & $3.1_{-0.6}^{+0.8}$ & 0.0124 & $52.0 \pm 23.0$ & 9 & -0.21 \\
\hline
\end{tabular}

Notes. ${ }^{(a)}$ Atmospheric parameters taken from Geier et al. (2011a). ${ }^{(b)}$ Atmospheric parameters derived from a spectrum taken with ESOVLT/FORS1. 